\title{
How is precision regulated in maintaining trunk posture?
}

\author{
Nienke W. Willigenburg $\cdot$ Idsart Kingma $\cdot$ \\ Jaap H. van Dieën
}

Received: 21 September 2009 / Accepted: 22 February 2010 / Published online: 14 March 2010

(C) The Author(s) 2010. This article is published with open access at Springerlink.com

\begin{abstract}
Precision of limb control is associated with increased joint stiffness caused by antagonistic co-activation. The aim of this study was to examine whether this strategy also applies to precision of trunk postural control. To this end, thirteen subjects performed static postural tasks, aiming at a target object with a cursor that responded to $2 \mathrm{D}$ trunk angles. By manipulating target dimensions, different levels of precision were imposed in the frontal and sagittal planes. Trunk angle and electromyography (EMG) of abdominal and back muscles were recorded. Repeated measures ANOVAs revealed significant effects of target dimensions on kinematic variability in both movement planes. Specifically, standard deviation (SD) of trunk angle decreased significantly when target size in the same direction decreased, regardless of the precision demands in the other direction. Thus, precision control of trunk posture was directionally specific. However, no consistent effect of precision demands was found on trunk muscle activity, when averaged over time series. Therefore, it was concluded that stiffness regulation by antagonistic co-activation was not used to meet increased precision demands in trunk postural control. Instead, results from additional analyses suggest that precision of trunk angle was controlled in a feedback mode.
\end{abstract}

N. W. Willigenburg · I. Kingma $\cdot$ J. H. van Dieën $(\bowtie)$

Faculty of Human Movement Sciences,

Research Institute MOVE, VU University Amsterdam,

Van der Boechorststraat 9, 1081 BT Amsterdam,

The Netherlands

e-mail: j.vandieen@fbw.vu.nl

N. W. Willigenburg

e-mail: n.willigenburg@fbw.vu.nl
Keywords Accuracy $\cdot$ Co-contraction $\cdot$ Stiffness $\cdot$ Spine . Motor control · Electromyography

\section{Introduction}

In controlling both posture and movement, regulation of joint stiffness is required to deal with perturbations. These perturbations can be either internal (van Galen and de Jong 1995) or external (forces applied to the body). With respect to internal perturbations, joint stiffness is associated with precision of motor control (Selen et al. 2005). Precision of motor control has been studied in the upper and lower limbs using several experimental tasks and outcome measures. In goal-directed arm movements, increased co-activation of antagonistic muscles (Gribble et al. 2003; Morishige et al. 2007; Osu et al. 2004) and increased elbow impedance (Selen et al. 2006a) coincided with increased precision demands. In arm tracking tasks, increased elbow impedance (Selen et al. 2006b) and increased co-activation of antagonistic forearm muscles (Huysmans 2008) were associated with increased precision demands. In the knee, increased quadriceps-to-hamstrings co-activation coincided with improved precision after learning to track a sinusoidal target during a resisted weight-bearing single-leg-squat (Madhavan and Shields 2009).

Stiffness regulation of the trunk has been studied in experiments with external perturbations. Lavender and colleagues reported anticipatory co-activation (feedforward) of abdominal and back muscles in expectance of an external load causing a flexion moment (Lavender et al. 1989). In lifting experiments, stiffening of the spine by antagonistic co-activation was reported when stability was challenged by increasing load height (Granata and Orishimo 2001) or introduction of variable load properties (van Dieën 
et al. 2003). However, there are also indications that the trunk neuromuscular system relies on feedback for the maintenance of postural control in dynamic loading conditions (Granata et al. 2001).

The human trunk is involved in almost all postural and movement tasks and it has a large number of degrees of freedom, since the spine consists of many joints. Furthermore, the spine is intrinsically unstable and, without active support, it will buckle under a load of approximately 90 Newton (Crisco et al. 1992). Thus, trunk muscle activity is necessary to control and stabilize the lumbar spine (Bergmark 1989). To our knowledge, precision control of trunk posture has never been studied. Since the mass of the trunk is larger than that of the limbs, the inertia is higher and thus the natural frequency is lower. By virtue of Newton's laws, this affects motor control by slowing the system. Furthermore, due to the complexity of the trunk musculature, which sub-serves multiple functions (e.g. posture and breathing), it is not evident that the same mechanism to regulate precision (stiffening by antagonistic co-activation) will be used in the trunk, as in the extremities. Surprisingly, the effect of increased precision demands on trunk stiffness has never been studied. Therefore, we developed experimental tasks imposing different levels of precision on trunk postural control, in order to investigate how this would affect trunk muscle activation and resulting kinematics.

Precision control in goal-directed arm movements is reported to be directionally specific. When aiming at targets with the same area, but a different shape (and thus different directional precision requirements), impedance was found to increase in the direction in which required precision was high (Lametti et al. 2007). Furthermore, in reaching movements to visual targets at the end of a visual track, the direction of greatest stiffness became oriented along the axis requiring greatest precision, thus perpendicular to the track (Wong et al. 2009b). In the trunk, indications for directionally specific stiffness regulation were also found in anticipation of postural perturbations (Allison et al. 2008; van Dieën and de Looze 1999). Whether this directional specificity also applies to precision control of trunk posture is unknown.

The main question in the present study was how, in absence of external perturbations, precision of trunk posture is regulated. Since internal perturbations due to neuromuscular noise are much more subtle than the externally applied forces in earlier studies on trunk postural control, it is not obvious that similar strategies will be used. By manipulating precision demands in the frontal and sagittal planes separately, it was studied to what extent trunk precision control is directionally specific. It was hypothesized that increased precision demands would result in a directionally specific reduction in trunk kinematic variability, associated with increased muscle co-activation.

\section{Methods}

Subjects

Thirteen healthy adults ( 8 male, 5 female, mean age $27.8 \pm 3.8$ years) participated after signing informed consent. Mean body length was $175 \pm 6.5 \mathrm{~cm}$ and mean body mass was $73.3 \pm 11.5 \mathrm{~kg}$.

\section{Experimental setup}

During the experiment, subjects maintained a semi-seated position, with their pelvis fixed in an adjustable frame and their arms crossed in front of their chest. A computer monitor was placed in front of the subjects (see Fig. 1). Real-time visual feedback of trunk angle (posture) was calculated from raw thorax marker and pelvis marker position data and presented on this monitor.

Up-down on the screen ( $y$-axis) corresponded with the sagittal plane angle between markers on thorax and pelvis (flexion/extension), and left-right on the screen ( $x$-axis) corresponded with the frontal plane angle (lateral flexion to the left and right). The screen was $600 \times 600$ pixels, with the $x$-axis from $-30^{\circ}$ (left) to $+30^{\circ}$ (right lateral flexion) and the $y$-axis from $-20^{\circ}$ (extension) to $40^{\circ}$ (flexion). Thus, in both the frontal and the sagittal planes, 1 pixel corresponded to $0.1^{\mathrm{o}}$ trunk angle.

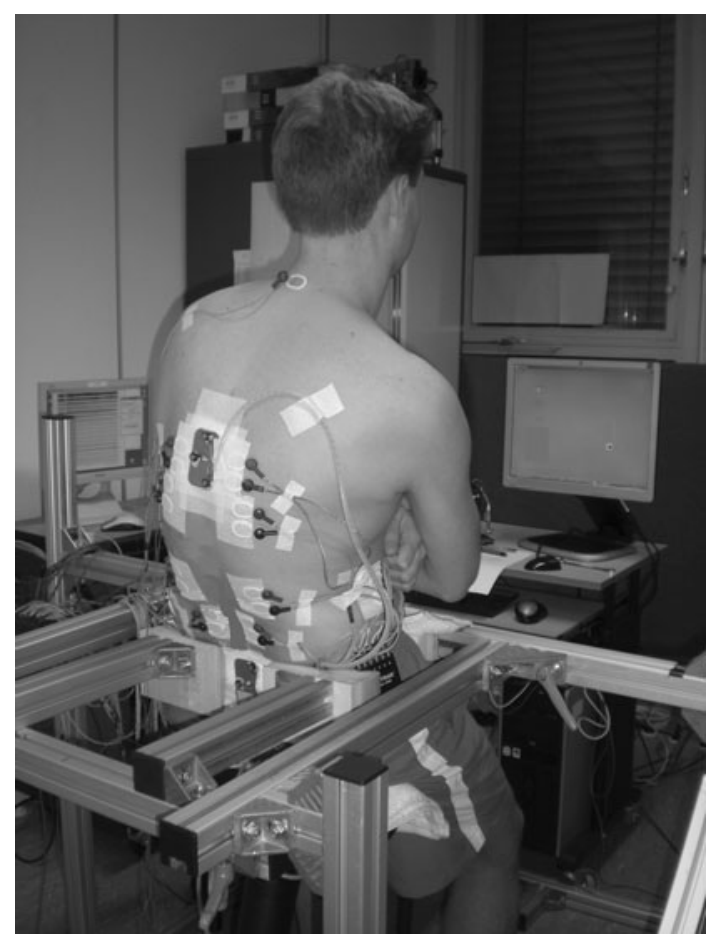

Fig. 1 Experimental setup 
Experimental tasks

At the beginning of each trial, real-time trunk angle feedback was projected on the gray screen as a black dot with a diameter of 20 pixels. In addition, a yellow target object was presented on the screen. Subjects were instructed to adjust their trunk posture in order to move to the target and then stay within this target. After five seconds, the black dot representing the real-time trunk angle disappeared and feedback was only presented when subjects left the target. When that happened, the side at which they crossed the target border turned red, indicating that the trunk angle had to be adjusted in the opposite direction.

By presenting the target object at different locations on the screen, five specific postures were imposed: the individually determined neutral posture $(\mathrm{N})$ and $20^{\circ}$ flexion (F), $10^{\circ}$ extension (E) and $10^{\circ}$ lateral flexion to the left (L) and to the right $(\mathrm{R})$ with respect to that neutral posture. By changing the dimensions of the target object, the required precision of trunk angle control in the frontal and sagittal plane was manipulated. Five different target dimensions were used: large square (lsq), medium square (msq), small square (ssq), horizontal rectangle (hor) and vertical rectangle (ver). With the small square, the margin in trunk angle was only $0.2^{\circ}$ from the target center in each direction. With the medium and the large square, the margins were $0.8^{\circ}$ and $1.4^{\circ}$, respectively. With the rectangular targets, the margin of the short side was $0.2^{\circ}$ and the margin of the long side was $1.4^{\circ}$, with the direction of these margins depending on the orientation of the target (horizontal or vertical).

The experimental protocol consisted of 20 trials ( 4 for each of the 5 target manipulations). Each trial contained 3 of the 5 postures as described previously and lasted approximately $2 \mathrm{~min}$. The target object always started in one of four 'non-neutral' positions and subjects maintained the corresponding posture for $35 \mathrm{~s}$. After that, the target moved in a straight line (with a bell-shaped velocity profile) and subjects tracked it to the neutral position, where they also stayed for $35 \mathrm{~s}$. Finally, the target moved and subjects tracked it to one of the other three positions (different from where it started), which they also maintained for $35 \mathrm{~s}$. Since the second posture of a trial was always the neutral posture, in 2 consecutive trials the postures $\mathrm{F}, \mathrm{E}, \mathrm{L}$ and $\mathrm{R}$ were performed once and the posture $\mathrm{N}$ was performed twice. The twelve $(4(\mathrm{~F} / \mathrm{E} /$ $\mathrm{L} / \mathrm{R}) \times 1(\mathrm{~N}) \times 3(\mathrm{~F} / \mathrm{E} / \mathrm{L} / \mathrm{R}-1))$ possible combinations of three postures within a trial and the order of trials were varied within and counterbalanced between subjects to avoid effects of fatigue. Between trials, subjects were allowed to take a short break whenever they wanted.
Procedure

After placing the EMG electrodes, isometric maximal voluntary contractions (MVCs) were performed for the different muscle groups, to normalize muscle activity during the experimental task. Subjects performed maximal exertions of flexion, extension and lateral flexion and rotation to both sides against the experimenters' manual resistance, while lying down.

After the MVC recordings, Optotrak markers were placed, and the experimental setup was adjusted to the subject. With fixed pelvis in the semi-seated posture, subjects were instructed to sit as comfortably as possible, in a neutral resting posture. The programmed trajectories of the target object were adapted to that resting posture and subjects performed two practice trials to familiarize with the experimental tasks. When the neutral posture felt incorrect (not neutral) during the practice trials, it was determined again. After familiarization with the tasks, subjects started with the first trial of the experimental protocol and after completion of all 20 trials, the markers and electrodes were removed.

\section{Data collection}

Two marker clusters, each containing three LED markers, were placed on the back; one on the thorax (T9 level) and one on the pelvis (S2 level). The 3D positions of these markers were recorded at a sample rate of 100 samples/s (Optotrak 3020, Northern Digital Inc, Canada; $\mathrm{SD} \approx 0.02 \mathrm{~mm}$ )

Electromyography (Porti 17, TMS, Enschede, The Netherlands; 22 bits AD conversion after $20 \times$ amplification, input impedance $>10^{12} \Omega$, CMRR $>90 \mathrm{~dB}$ ) of four abdominal and four back muscles was measured, both left and right. Therefore, pairs of surface EMG electrodes (Ag/ $\mathrm{AgCl}$, inter-electrode distance $25 \mathrm{~mm}$ ) were attached to the skin after shaving and cleaning with alcohol. On the back, electrodes were placed $4 \mathrm{~cm}$ lateral to $\mathrm{T} 9$ spinous process [thoracic part of $\mathrm{m}$. longissimus (LT)], $6 \mathrm{~cm}$ lateral to T11 [thoracic part of m. iliocostalis (IT)] and L2 [lumbar part of $\mathrm{m}$. iliocostalis (IL)] spinous processes and $3 \mathrm{~cm}$ lateral to the midpoint between the spinous processes of L3 and L4 [lumbar part of $\mathrm{m}$. longissimus (LL)]. To measure abdominal muscle activity, electrodes were placed $3 \mathrm{~cm}$ lateral to the umbilicus or somewhat lower when a tendinous intersection was present there [m. rectus abdominus (RA)], $3 \mathrm{~cm}$ medial to the anterior superior iliac spine (ASIS) (m. obliquus internus (OI)), in the mid-axillary line between the iliac crest and the 10th rib (lateral part of $\mathrm{m}$. obliquus externus (OEL)) and at the crossing point of a horizontal line through the umbilicus and a vertical line through the ASIS (anterior part of $\mathrm{m}$. obliquus externus (OEA)). 
Muscle activity was recorded at a sample rate of 1,000 sample/s, and a pulse signal was used to synchronize the kinematic and EMG data.

\section{Data analysis}

All data of experimental trials were split, in that three 30-s time series were selected for each posture. Transition periods, when subjects moved from one posture to the other, and the first $5 \mathrm{~s}$ in each new posture were not further analyzed. In total, for each of the five target dimensions, 4 time series of $30 \mathrm{~s}$ in the neutral posture and 2 time series of $30 \mathrm{~s}$ in the four other postures were analyzed for each subject.

Kinematic data of the highest (thorax) and lowest (pelvis) marker were filtered (1 Hz low pass, 4th order bi-directional Butterworth) and trunk angles relative to the vertical were calculated in the frontal and sagittal plane. The target angle was subtracted from the actual trunk angle and the standard deviation (SD) of the resulting angular deviations was calculated as a measure of kinematic variability. In addition, spectral analysis (Welch's averaged periodogram method, window size $15 \mathrm{~s}$ with $70 \%$ overlap between consecutive windows to enhance low frequency detection) of the trunk angle time series was performed to calculate the mean power frequency (MPF). This mean power frequency depends on the frequency of postural adjustments and was expected to increase with increased precision demands.

To remove the $50-\mathrm{Hz}$ interference that was found in some EMG signals, a 49.5 to $50.5-\mathrm{Hz}$ band stop filter (4th order bi-directional Butterworth) was applied to the EMG data. After that, ECG contamination was removed using independent component analysis, using the FastICA algorithm (Hyvärinen and Oja 2000) and a 10 to $400-\mathrm{Hz}$ band pass filter (4th order bi-directional Butterworth) was applied. Subsequently, the signals were rectified and a 2.5-Hz low-pass filter (2nd order uni-directional Butterworth) was applied to correct for electromechanical delay (Brereton and McGill 1998; Potvin et al. 1996). Finally, a bi-directional low-pass filter with a cutoff frequency of $1 \mathrm{~Hz}$, equal to the frequency below which $95 \%$ of the power occurred in the $(5 \mathrm{~Hz}$ low pass filtered) kinematic signal, was applied in order to obtain optimal matching between muscle activity and trunk angle time series. The resulting EMG linear envelopes formed the starting point for calculating the following EMG outcome measures.

After normalization to MVC, mean and SD of EMG amplitudes were calculated for each muscle. By dividing SD by mean amplitude, the coefficient of variation (COV) was calculated as well. To obtain the same variables for the four muscle groups (abdominal, back, left and right), the 16 muscles were grouped either as 8 abdominal and 8 back muscles, or as 8 left and 8 right muscles, and their values were averaged. Ratios of co-activation were calculated by dividing antagonist by agonist muscle group mean amplitude. In addition, spectral analysis of EMG linear envelopes (Welch's averaged periodogram method, window size $15 \mathrm{~s}$ with $70 \%$ overlap between consecutive windows to enhance low frequency detection) was performed to calculate the MPF. These MPF of EMG linear envelopes were expected to increase with increased precision demands, similar to the MPF of kinematic time series.

\section{Statistics}

SPSS 16.0 was used for statistical analysis. Both kinematic (SD and MPF of trunk angles in frontal and sagittal planes) and EMG data (mean amplitude, SD, COV and MPF for each muscle and muscle group and ratio of co-activation) were analyzed using 5 (target: hor, ver, lsq, msq, ssq) $\times 5$ (trunk posture: $\mathrm{F}, \mathrm{E}, \mathrm{L}, \mathrm{R}, \mathrm{N}$ ) ANOVAs with Bonferroni post hoc corrections. The level of significance was set at $\alpha<0.05$, and Greenhouse-Geisser corrections were used when the assumption of sphericity was violated.

\section{Results}

Figure 2 shows typical time series and power spectra of a subject performing one experimental trial, starting in extension, then tracking to the neutral posture and finally tracking to flexion.

\section{Task performance}

Percentages of time on target, averaged over postures and subjects, are shown in Table 1. Repeated measures ANOVA revealed a significant main effect of target $(P<0.001)$. Pairwise comparisons indicated that all percentages differed significantly from each other $(P<0.02)$, except that the percentage time on target for the vertical target was not different from that for the horizontal and the small square target.

\section{Effect of target dimension on kinematic variability}

Precision demands affected trunk postural control as hypothesized, since repeated measures ANOVAs revealed a main effect of target dimensions on the SD of trunk angle time series (Fig. 3), both in the frontal and sagittal planes $(P<0.001)$. No target $\times$ posture interaction effect was found (both $P \geq 0.122$ ).

When considering square targets only, pairwise comparisons indicated that trunk angle SDs decreased in both the frontal and sagittal planes with decreasing target size (Table 2). All differences were significant, except for the 
Kinematics in frontal (dotted) and sagittal (solid) planes
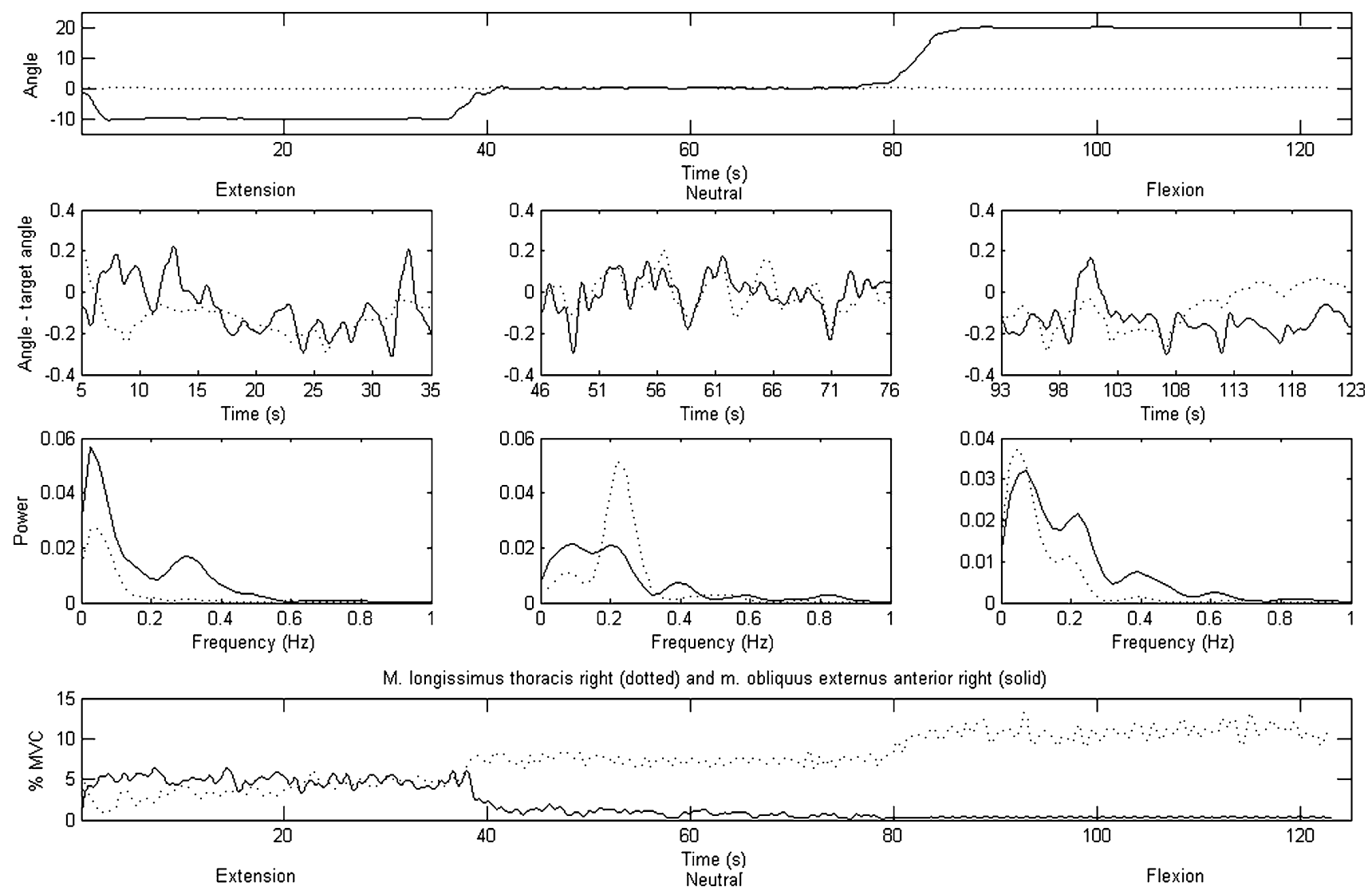
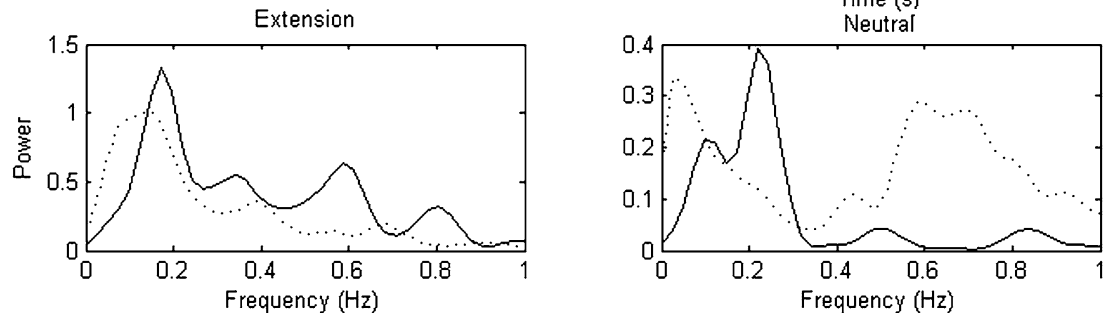

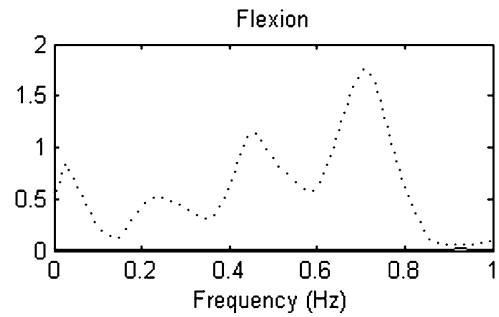

Fig. 2 Typical example of kinematic and EMG data, from subject 8 performing a trial starting in $10^{\circ}$ extension and ending in $20^{\circ}$ flexion (with a small square target). In the upper panel, trunk angle over the complete trial is shown for both planes of motion. In the second panel, the selected time series are shown, with target angle subtracted from actual angle and transition periods removed. The third panel shows the

Table 1 Percentages of time on target

\begin{tabular}{ll}
\hline Target & Score $($ mean \pm SD) \\
\hline hor & $89.7 \pm 3.8$ \\
ver & $89.6 \pm 4.3$ \\
lsq & $95.3 \pm 2.6$ \\
msq & $94.2 \pm 2.8$ \\
ssq & $86.1 \pm 4.7$ \\
\hline
\end{tabular}

difference between the large and medium square targets in the frontal plane.

When considering all targets, pairwise comparisons also revealed direction-specific effects of target dimensions on power spectra for all three postures within this trial. The fourth panel shows EMG linear envelopes of one abdominal and one back muscle. In the lower panel, power spectra of these EMG linear envelopes are shown. Large variations in power distributions were found within and between subjects. Note that the activity of the right $\mathrm{m}$. obliquus externus anterior is very low in flexion, resulting in almost zero power

trunk angle SD (Table 2). In both planes, SDs were significantly smaller when the target size was smaller and this did not depend on the precision demands in the other plane. SDs for targets with the same size in one of the directions did not differ from each other in that direction. Averaged over postures, SDs were $0.27 \pm 0.04^{\circ}$ (sagittal plane) and $0.19 \pm 0.05^{\circ}$ (frontal plane) with the lowest precision demands (margin $1.4^{\circ}$ ) and $0.12 \pm 0.03^{\circ}$ (sagittal plane) and $0.10 \pm 0.02^{\circ}$ (frontal plane) with the highest precision demands (margin $0.2^{\circ}$ ).

Repeated measures ANOVAs also revealed a main effect of target dimensions on the MPF of trunk angles in both movement planes $(P<0.001)$. Pairwise comparisons 
Fig. 3 SDs of trunk angle time series, in the frontal and sagittal planes for all trunk postures. The five bars represent the different targets (horizontal, vertical, large square, medium square and small square, respectively)
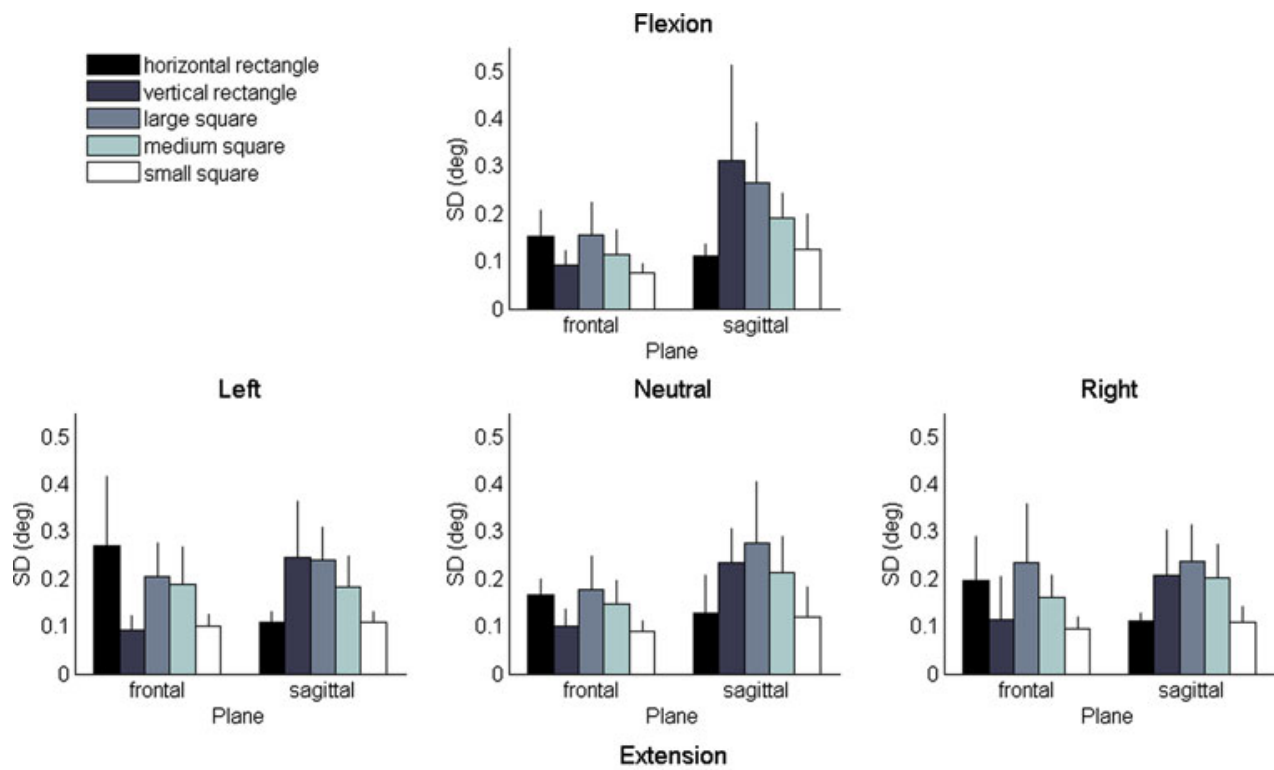

Table $2 P$-values of pairwise comparisons for the main effect of target dimensions on trunk angle SDs in the sagittal (S) and frontal (F) planes in the left lower and the right upper part of the table, respectively

\begin{tabular}{llllll}
\hline Target & hor & ver & lsq & msq & ssq \\
\hline hor & & $<0.001$ & 1.000 & 0.007 & $<0.001$ \\
ver & $<0.001$ & & $<0.001$ & $<0.001$ & 1.000 \\
lsq & $<0.001$ & 1.000 & & 0.054 & $<0.001$ \\
msq & $<0.001$ & 0.003 & $<0.001$ & & $<0.001$ \\
ssq & 1.000 & $<0.001$ & $<0.001$ & $<0.001$ & \\
\hline
\end{tabular}

$P$-values in italics are significant $(<0.05)$

(Table 3) indicated a higher MPF with higher precision demands for each direction, but results were less consistent than for SD. No target $\times$ posture interaction effect was found on MPF of trunk angle time series (both $P \geq 0.400$ ).

\section{Effect of target dimension on EMG}

In contrast with the hypothesis, repeated measures ANOVAs did not reveal a significant main effect of target dimensions on EMG amplitudes averaged within muscle groups (Fig. 4; abdominal: $P=0.226$, back: $P=0.899$, left: $P=0.100$ and right: $P=0.583$ ), and anta/agonist ratio $(P=0.367)$. When analyzing muscles separately (Fig. 5),

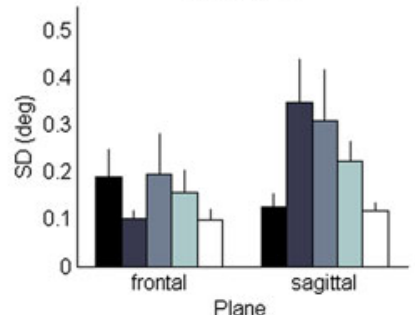

Table 3 Mean power frequencies (Hz) of trunk angle in both movement planes for all target dimensions

\begin{tabular}{lll}
\hline Plane target & MPF sagittal $(\mathrm{Hz})$ & MPF frontal $(\mathrm{Hz})$ \\
\hline hor & $0.221 *$ ver,lsq,msq & $0.137 *$ ver,ssq \\
ver & $0.134 *$ hor,ssq & $0.187 *$ hor,lsq,msq \\
lsq & $0.134 *$ hor,ssq & $0.13 *^{*}$ ver,ssq \\
msq & $0.139 *$ hor,ssq & $0.143 *$ ver,ssq \\
ssq & $0.228 *$ ver,lsq,msq & $0.191 *$ hor,lsq,msq \\
\hline
\end{tabular}

Significant differences between target dimensions are shown in superscript

no main effect of target was found in mean amplitude (all $P \geq 0.070)$, except for one muscle: OELL $(P=0.035)$. However, this effect was small and pairwise comparisons indicated only one significant difference between two targets $(14.9 \%$ higher amplitude in OELL with small (2.622\% MVC) compared to large (2.282\%MVC) square target). No significant main effect of target dimensions was found on SD (all $P \geq 0.294$ ), COV (all $P \geq 0.462$ ) and MPF (all $P \geq 0.291$ ) of averaged EMG linear envelopes within muscle groups or on SD (all $P \geq 0.129$ ), COV (all $P \geq 0.077$, except for ILL: $P=0.036$ ) and MPF (all $P \geq 0.135$, except for ILR: $P=0.029)$ of EMG linear envelopes of separate muscles. No target $\times$ posture interaction was found on any of the EMG variables. 
Fig. 4 Agonist and antagonist activity (\% MVC) for the different trunk postures, with the five bars representing different targets (horizontal, vertical, large square, medium square and small square, respectively). No consistent effect of target was found, and the error bars indicate large inter-individual differences
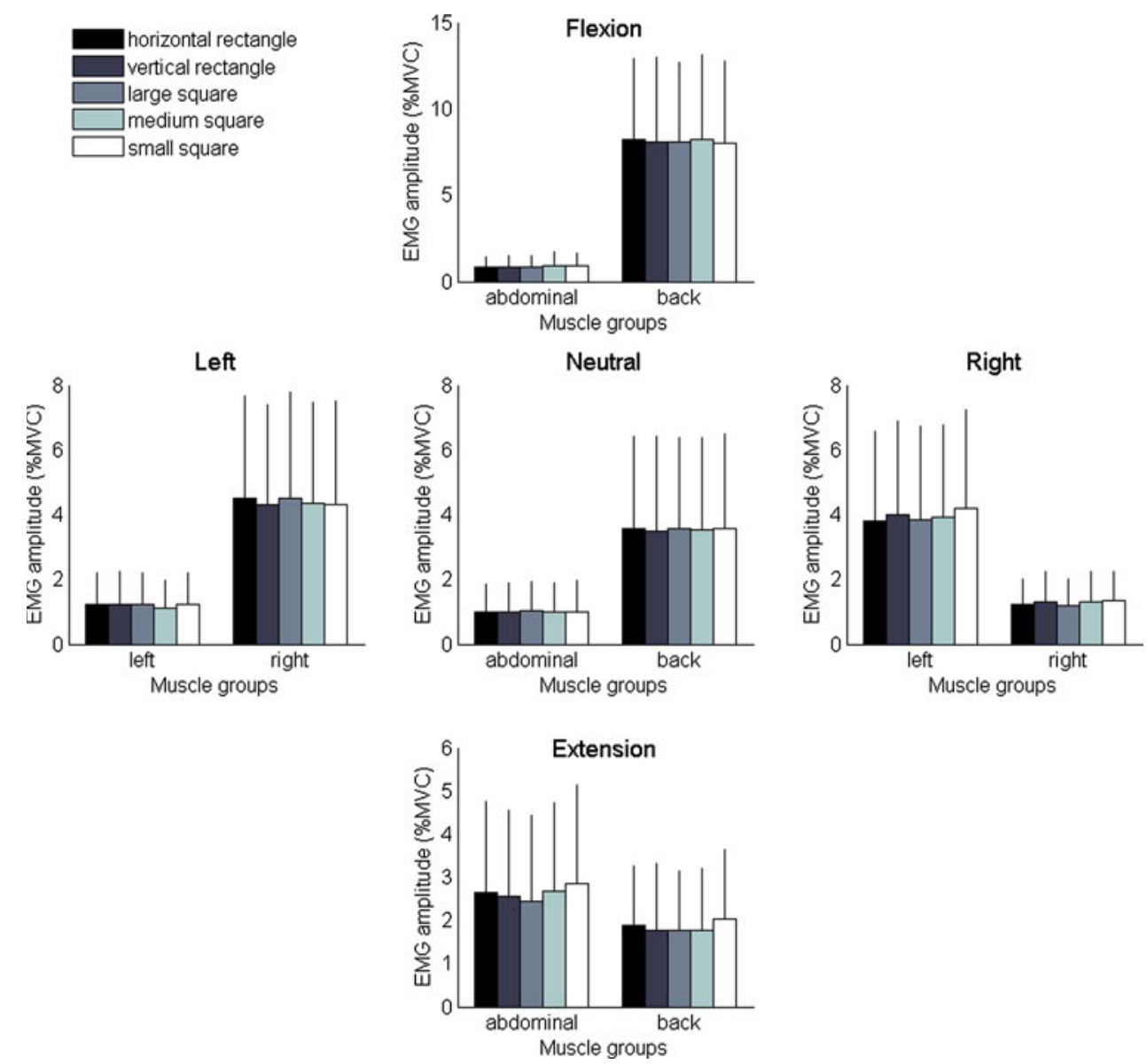

target dimensions on EMG amplitude, SD, COV and MPF was found. Since no feedforward co-activation of trunk muscles was found with increased precision demands, subjects may have used a feedback strategy to control their trunk posture. To find support for this post hoc hypothesis, additional analyses were performed in order to relate kinematics and EMG in time. For EMG time series, a combined measure for all muscles working in one movement plane was calculated. For the sagittal plane, EMG linear envelopes averaged over all eight abdominal muscles were subtracted from EMG linear envelopes averaged over all eight back muscles, thus $\mathrm{EMG}_{\mathrm{sag}}=\mathrm{EMG}_{\mathrm{back}}-\mathrm{EMG}_{\mathrm{abd}}$. For the frontal plane, EMG linear envelopes averaged over all eight right muscles were subtracted from EMG linear envelopes averaged over all eight left muscles, thus $\mathrm{EMG}_{\text {front }}=\mathrm{EMG}_{\text {left }}-\mathrm{EMG}_{\text {right }}$. Subtraction was used rather than addition, since alternating activity of opposing muscle groups is expected to cause kinematic variation, whereas simultaneous activity of opposing muscle groups is not. When considering the axes definitions in the present study, positive correlations between $\mathrm{EMG}_{\mathrm{sag}}$ and sagittal plane kinematics and between $\mathrm{EMG}_{\text {front }}$ and frontal plane kinematics were expected. Indeed, in the frontal plane, a correlation coefficient significantly larger than zero was found for all target dimensions $\left(r_{\text {hor }}=0.170 \pm 0.092\right.$, increasing precision constraints, no consistent effect of 
Fig. 5 Mean activity (\% MVC) averaged over postures for each muscle, with the five bars representing different targets (horizontal, vertical, large square, medium square and small square, respectively). No consistent effect of target was found, and the error bars indicate large inter-individual differences

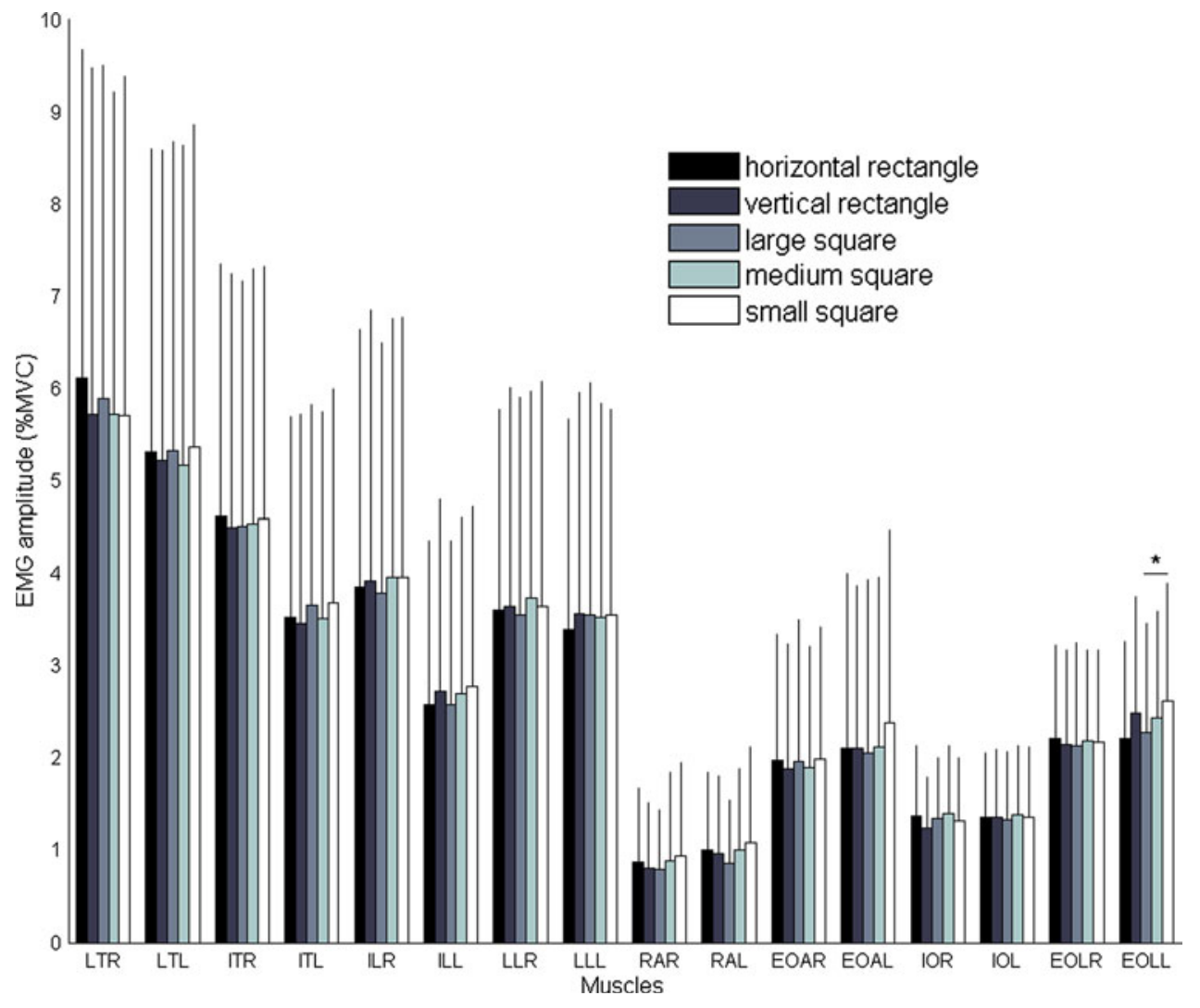

$\mathrm{r}_{\mathrm{ver}}=0.128 \pm 0.075, \quad \mathrm{r}_{\mathrm{lsq}}=0.142 \pm 0.072, \quad \mathrm{r}_{\mathrm{msq}}=0.161 \pm$ $0.093, \mathrm{r}_{\mathrm{ssq}}=0.094 \pm 0.085$, all $\left.P \leq 0.002\right)$. In the sagittal plane, a correlation coefficient significantly larger than zero was found only for the targets with lowest precision demands in that plane $\left(\mathrm{r}_{\mathrm{ver}}=0.174 \pm 0.091, \mathrm{r}_{\mathrm{lsq}}=0.187 \pm\right.$ 0.128 , both $P \leq 0.001)$. Although these correlation coefficients were not particularly high, this implied that kinematics and EMG activity were related, at least in the direction with low precision demands. Note that the smallest margin $\left(0.2^{\circ}\right.$ from the target center) corresponded with $\pm 0.1 \mathrm{~cm}$ displacement of the center of mass of the upper body (trunk, head and arms). Presume an upper body mass of $35 \mathrm{~kg}$, than this displacement would induce a $0.35-\mathrm{Nm}$ change in moment around the L5/S1 segment and the muscle activity needed for such subtle corrections is very small. Together with a larger SD in the sagittal plane kinematics, this could explain the low correlations when precision demands were high.

Thus, in the direction where precision demands were high, variations in posture were smaller, which hindered the detection of changes in EMG. With high precision demands, subjects received more visual feedback during each trial, since they left the target more often. Averaged over postures, subjects crossed the target border $8.6 \pm 2.1$ times with the small square target, versus $0.8 \pm 0.5$ times with the large square target during the 30-s time series. So, visual feedback appeared roughly 10 times more often when precision demands were high. To quantify the effect of this visual feedback, EMG amplitudes during a short period $(0.5 \mathrm{~s})$ before and after the appearance of visual feedback were compared. A 50-ms delay was included to account for the time between a simple stimulus and a detectable change in trunk muscle EMG activity, based on fast $(110 \mathrm{~ms})$ kinematic responses of the human hand (Brenner and Smeets 1997), roughly corrected for electromechanical delay and the distance from central nervous system to neuromuscular junction. The appropriate response to the appearance of visual feedback was increased activity in the direction opposite to the side of the target that turned red. For instance, when crossing the border of a target at the lower side, trunk posture should be corrected more toward flexion, so the value of $\mathrm{EMG}_{\mathrm{sag}}$ (back-abdominal muscle EMG) is expected to decrease from pre- to post-feedback appearance. By subtracting the expected lower value from the expected higher value of EMG in the plane of motion concerned (e.g. post-pre $\mathrm{EMG}_{\text {sag }}$ in response to visual feedback at the upper side and pre-post $E M G_{\text {fro }}$ in response to visual feedback at the left side of a target), a resulting value significantly larger than zero $(0.06 \pm 0.04 \% \mathrm{MVC}, P<0.001)$ remained after averaging over targets and postures. This indicates that subjects indeed increased muscle activity opposite to the side where visual feedback appeared, as expected for successfully adjusting trunk posture. The fact that the magnitude of this 
effect was quite small indicates that the adjustments were very subtle. This was not surprising, since the margins were so small that the risk of over-adjusting was high.

\section{Discussion}

In the present study, required precision of trunk postural control was manipulated by changing the dimensions of a target object. Kinematic variability was significantly affected by target dimensions, in that for the square targets, SD of trunk angle decreased significantly with decreasing target size. For the rectangular targets, SD in the direction of the short side was similar to that with the small square target and significantly lower compared to that in the direction of the long side and to those with the medium and large square target. Apparently, precision of trunk posture was controlled directionally specific, with SDs for the highest precision demands being half of the SDs for the lowest precision demands. The slightly higher SDs in the sagittal plane when compared to the frontal plane might be due to effects of respiration (Grimstone and Hodges 2003; Hodges et al. 2002) or to the symmetry of muscles in the frontal plane versus the asymmetry of muscles in the sagittal plane which could affect precision control.

Despite these effects on kinematic variability, no consistent effects of precision demands were found on trunk muscle activity averaged over time series, since none of the EMG variables (mean amplitude, SD, COV and ratio of coactivation) was substantially affected by target dimensions. The observed significant effect on OELL amplitude may be a type I error and cannot solely explain the consistent effects observed in the kinematic data. The absence of effects of target dimensions on trunk muscle group EMG amplitude and anta/agonist ratio implies that stiffness modulation by antagonistic co-activation of superficial trunk muscles was not used to limit kinematic variability of the trunk angle.

Therefore the question remained how subjects managed to improve precision when this was required. Two alternative explanations can be proposed to answer this question. First, it is possible that a feedback strategy was used rather than a feedforward co-activation strategy, since co-activation is metabolically less efficient. Additional analyses provided some evidence for such a feedback strategy. Second, it is possible that stiffness regulation occurred by co-activation of deep trunk muscles such as $\mathrm{m}$. transversus abdominis and mm. multifidi. No intramuscular EMG was measured and thus no information was available on the activity of these muscles. Both these alternative explanations will be discussed in some more detail.

The appropriate responses to the appearance of visual feedback (when subjects crossed the target border) provided evidence for feedback rather than feedforward control of trunk posture. Specifically (relative) muscle activity opposite to the side where visual feedback appeared increased, implying postural adjustments in the correct direction. Thus, instead of stiffening the trunk by antagonistic co-activation to prevent crossing the target borders, it appeared that subjects waited until they felt (proprioception) or saw (visual feedback) that it was necessary to adjust their posture, implying feedback control. Within this explanation based on feedback control, deep trunk muscles are likely to be involved as well. Probably, visual feedback was the dominant source of feedback when precision demands were high. With a small square target, visual feedback appeared 10 times more often when compared to a large square target. When precision demands were low and the greater margin allowed more variation in trunk angle, proprioception possibly became a more important source of feedback.

The possibility that co-activation of deep trunk muscles occurred cannot be excluded by the current study. Support for this alternative explanation for reduced kinematic variability is that these muscles appear to have a special role in the control of trunk posture. For instance, m. transversus abdominis and the deep fibers of $\mathrm{mm}$. multifidi are both active in a non-direction-specific feedforward manner in preparation for the perturbation to the spine induced by rapid arm movements (Hodges and Richardson 1997; Moseley et al. 2002). However, modeling studies suggest that their stabilizing potential would be limited (Crisco and Panjabi 1991) or at least not larger than that of other trunk muscles (Cholewicki and VanVliet 2002). Furthermore, although intra-muscular EMG recordings are recommended to measure activity of these deep muscles, it has been shown that part of their activity is detected by surface electrodes at locations comparable to the ones used in this study (Marshall and Murphy 2003; McGill et al. 1996; Stokes et al. 2003). Therefore, if substantial co-activation occurred in deep muscles, we might have found increased amplitudes in the overlying muscles' sEMG. Finally, increased co-activation of superficial muscles to increase trunk stiffness was found in previous studies recording surface EMG (Granata and Orishimo 2001; van Dieën et al. 2003). Although external perturbations were applied in these studies, this implies that superficial muscles are involved in trunk stiffness regulation.

Since no antagonistic co-activation was found, the current study implies that neither previous findings on precision control in the limbs (Gribble et al. 2003; Huysmans 2008; Lametti et al. 2007; Madhavan and Shields 2009; Morishige et al. 2007; Osu et al. 2004; Selen et al. 2006a; Selen et al. 2006b; Wong et al. 2009a) nor previous findings in studies with external perturbations applied to the trunk (Allison et al. 2008; Granata et al. 2001; van Dieën 
and de Looze 1999; van Dieën et al. 2003) can be generalized to precision control of the trunk. The current study does support the conclusion of Granata and colleagues that the trunk neuromuscular system relies on feedback for the maintenance of postural control (Granata et al. 2001). The use of feedback control seems plausible, since the observed range of movement frequencies (mean power frequencies varied from 0.13 to $0.23 \mathrm{~Hz}$ ) is indeed low enough to be adequately controlled by feedback, in spite of the delays involved (Goodworth and Peterka 2009).

The absence of continuous feedback of trunk angle (feedback was only provided when subjects left the target) could be suggested as a limitation of this study. However, a pilot study with continuous feedback (unpublished data) resulted in the same findings. It was expected that continuous feedback facilitated a feedback control strategy and therefore the current methods were developed to promote a feedforward control strategy. However, even the current results failed to demonstrate feedforward co-activation of superficial trunk muscles to increase precision of trunk postural control.

Another possible limitation is that subjects could achieve the imposed trunk angle in different ways, due to the multiple degrees of freedom in the lumbar spine. Previous studies also found that specific target positions and orientations of trunk markers can be achieved with different levels of lumbar lordosis (Claus et al. 2009; Kingma et al. 2007). Furthermore, only data-series of 30-s static postural tasks were analyzed. Therefore, it cannot be excluded that coactivation strategies would be used in either more restricted or more dynamic postural tasks. In conclusion, the present study provided no evidence for stiffness regulation through co-activation of superficial trunk muscles in precision control of trunk posture. Instead, additional analyses suggest that precision of trunk angle was controlled in a feedback mode.

Open Access This article is distributed under the terms of the Creative Commons Attribution Noncommercial License which permits any noncommercial use, distribution, and reproduction in any medium, provided the original author(s) and source are credited.

\section{References}

Allison GT, Morris SL, Lay B (2008) Feedforward responses of transversus abdominis are directionally specific and act asymmetrically: implications for core stability theories. J Orthop Sports Phys Ther 38:228-237

Bergmark A (1989) Stability of the lumbar spine. A study in mechanical engineering. Acta Orthop Scand Suppl 230:1-54

Brenner E, Smeets JB (1997) Fast responses of the human hand to changes in target position. J Mot Behav 29:297-310

Brereton LC, McGill SM (1998) Frequency response of spine extensors during rapid isometric contractions: effects of muscle length and tension. J Electromyogr Kinesiol 8:227-232
Cholewicki J, VanVliet JJ (2002) Relative contribution of trunk muscles to the stability of the lumbar spine during isometric exertions. Clin Biomech 17:99-105

Claus AP, Hides JA, Moseley GL, Hodges PW (2009) Different ways to balance the spine: subtle changes in sagittal spinal curves affect regional muscle activity. Spine 34:E208-E214

Crisco JJ, Panjabi MM (1991) The intersegmental and multisegmental muscles of the lumbar spine - a biomechanical model comparing lateral stabilizing potential. Spine 16:793-799

Crisco JJ, Panjabi MM, Yamamoto I, Oxland TR (1992) Euler stability of the human ligamentous lumbar spine. Part II: Experiment. Clin Biomech 7:27-32

Goodworth AD, Peterka RJ (2009) Contribution of sensorimotor integration to spinal stabilization in humans. J Neurophysiol 102:496-512

Granata KP, Orishimo KF (2001) Response of trunk muscle coactivation to changes in spinal stability. J Biomech 34:1117-1123

Granata KP, Orishimo KF, Sanford AH (2001) Trunk muscle coactivation in preparation for sudden load. J Electromyogr Kinesiol 11:247-254

Gribble PL, Mullin LI, Cothros N, Mattar A (2003) Role of co-contraction in arm movement accuracy. J Neurophysiol 89:2396-2405

Grimstone S, Hodges P (2003) Impaired postural compensation for respiration in people with recurrent low back pain. Exp Brain Res 151:218-224

Hodges PW, Richardson CA (1997) Feedforward contraction of transversus abdominis is not influenced by the direction of arm movement. Exp Brain Res 114:362-370

Hodges P, Gurfinkel V, Brumagne S, Smith T, Cordo P (2002) Coexistence of stability and mobility in postural control: evidence from postural compensation for respiration. Exp Brain Res 144:293302

Huysmans MA (2008) Sub-movement organisation and impedance are modulated in response to precision demands in 2D tracking. In: From precision demands to neck and upper extremity pain $(\mathrm{PhD}$ thesis), VU University Amsterdam, pp 39-57

Hyvärinen A, Oja E (2000) Independent component analysis: algorithms and applications. Neural Netw 13:411-430

Kingma I, Staudenmann D, van Dieën JH (2007) Trunk muscle activation and associated lumbar spine joint shear forces under different levels of external forward force applied to the trunk. J Electromyogr Kinesiol 17:14-24

Lametti DR, Houle G, Ostry DJ (2007) Control of movement variability and the regulation of limb impedance. $J$ Neurophysiol 98:3516-3524

Lavender SA, Mirka GA, Schoenmarklin RW, Sommerich CM, Sudhakar LR, Marras WS (1989) The effects of preview and task symmetry on trunk muscle response to sudden loading. Hum Factors 31:101-115

Madhavan S, Shields RK (2009) Movement accuracy changes muscleactivation strategies in female subjects during a novel single-leg weight-bearing task. Pm R 1:319-328

Marshall P, Murphy B (2003) The validity and reliability of surface EMG to assess the neuromuscular response of the abdominal muscles to rapid limb movement. J Electromyogr Kinesiol 13:477-489

McGill S, Juker D, Kropf P (1996) Appropriately placed surface EMG electrodes reflect deep muscle activity (psoas, quadratus lumborum, abdominal wall) in the lumbar spine. J Biomech 29:15031507

Morishige K-i, Osu R, Kamimura N, Iwasaki H, Miyamoto H, Wada Y, Kawato M (2007) How can we realize skillful and precise movement? Int Congr Ser 1301:188-191

Moseley GL, Hodges PW, Gandevia SC (2002) Deep and superficial fibers of the lumbar multifidus muscle are differentially active during voluntary arm movements. Spine 27:E29-E36 
Osu R, Kamimura N, Iwasaki H, Nakano E, Harris CM, Wada Y, Kawato M (2004) Optimal impedance control for task achievement in the presence of signal-dependent noise. J Neurophysiol 92:1199-1215

Potvin J, Norman R, McGill S (1996) Mechanically corrected EMG for the continuous estimation of erector spinae muscle loading during repetitive lifting. Eur J App Physiol O 74:119-132

Selen LP, Beek PJ, van Dieën JH (2005) Can co-activation reduce kinematic variability? A simulation study. Biol Cybern 93:373-381

Selen LP, Beek PJ, van Dieën JH (2006a) Impedance is modulated to meet accuracy demands during goal-directed arm movements. Exp Brain Res 172:129-138

Selen LP, van Dieën JH, Beek PJ (2006b) Impedance modulation and feedback corrections in tracking targets of variable size and frequency. J Neurophysiol 96:2750-2759

Stokes IA, Henry SM, Single RM (2003) Surface EMG electrodes do not accurately record from lumbar multifidus muscles. Clin Biomech (Bristol, Avon) 18:9-13 van Dieën JH, de Looze MP (1999) Directionality of anticipatory activation of trunk muscles in a lifting task depends on load knowledge. Exp Brain Res 128:397-404

van Dieën JH, Kingma I, van der Burg P (2003) Evidence for a role of antagonistic cocontraction in controlling trunk stiffness during lifting. J Biomech 36:1829-1836

van Galen GP, de Jong WP (1995) Fitts' law as the outcome of a dynamic noise filtering model of motor control. Hum Mov Sci 14:539-571

Wong J, Wilson ET, Malfait N, Gribble PL (2009a) The influence of visual perturbations on the neural control of limb stiffness. J Neurophysiol 101:246-257

Wong J, Wilson ET, Malfait N, Gribble PL (2009b) Limb stiffness is modulated with spatial accuracy requirements during movement in the absence of destabilizing forces. J Neurophysiol 101:15421549 